\title{
Organização do atendimento em saúde durante pandemia do coronavírus: o reflexo do trabalho colaborativo
}

Organization of health care during a coronavirus pandemic: the reflection of collaborative work Organización de la asistencia sanitaria durante una pandemia de coronavirus: el reflejo del trabajo colaborativo

\begin{abstract}
RESUMO
Apesar das mudanças trazidas pela COVID-19, a atenção primária à saúde precisa assegurar o acesso e cobertura do território de forma integral e universal. Objetivo: Relatar a experiência de uma residente em saúde da familia acerca da reorganização do fluxo de atendimento em uma unidade de saúde da familia durante a pandemia do Coronavírus. Método: Trata-se de um estudo descritivo, do tipo relato de experiência, acerca da vivência de uma residente inserida na estratégia de saúde da famîlia da unidade José Adelino da Silva, no município de Porto Velho, Rondônia. Resultados: 0 fluxo de cuidado foi estruturado visando reduzir aglomerações e manter o acesso e resolutividade. Organizou-se as demandas espontâneas, programadas e procedimentos da carteira de serviço da USF. Conclusão: A reorganização dos fluxos de atendimento evidenciou a potencialidade do trabalho colaborativo. Foi possivel implantar o acolhimento, escuta qualificada, agenda programada e não foram observados aglomerações e filas.
\end{abstract}

DESCRITORES: Infecções por Coronavirus; Atenção Primária à Saúde; Planejamento em saúde. Práticas interdisciplinares.

\section{ABSTRACT}

Despite the changes brought about by COVID-19, primary health care needs to ensure access and coverage of the territory in a comprehensive and universal manner. Objective: To report the experience of a family health resident about the reorganization of the flow of care in a family health unit during the Coronavirus pandemic. Method: This is a descriptive study, of the experience report type, about the experience of a resident inserted in the family health strategy of the José Adelino da Silva unit, in the city of Porto Velho, Rondônia. Results: The care flow was structured in order to reduce agglomerations and maintain access and resolution. The spontaneous, programmed demands and procedures of the USF service portfolio were organized. Conclusion: The reorganization of the service flows showed the potential of collaborative work. It was possible to implement the reception, qualified listening, programmed agenda and no agglomerations and queues were observed.

DESCRIPTORS: Coronavirus infections; Primary Health Care; Health planning. Interdisciplinary practices.

\section{RESUMEN}

A pesar de los cambios provocados por el COVID-19, la atención primaria de salud necesita asegurar el acceso y cobertura del territorio de manera integral y universal. Objetivo: Informar la experiencia de un residente de salud familiar sobre la reorganización del flujo de atención en una unidad de salud familiar durante la pandemia de Coronavirus. Método: Se trata de un estudio descriptivo, del tipo relato de experiencia, sobre la experiencia de un residente inserto en la estrategia de salud familiar de la unidad José Adelino da Silva, en la ciudad de Porto Velho, Rondônia. Resultados: El flujo de atención se estructuró con el fin de reducir las aglomeraciones y mantener el acceso y la resolución. Se organizaron las demandas y procedimientos espontáneos y programados de la cartera de servicios de la USF. Conclusión: La reorganización de los flujos de servicios mostró el potencial del trabajo colaborativo. Se logró implementar la recepción, escucha calificada, agenda programada y no se observaron aglomeraciones y colas.

DESCRIPTORES: Infecciones por Coronavirus; Atención Primaria de Salud. Planificación sanitária. Practicas interdisciplinariais.

RECEBIDO EM: 24/03/2021 APROVADO EM: 24/05/2021

\section{Maria Juliana Vasconcellos Bragado}

Fisioterapeuta, pós-graduanda da Residência Multiprofissional em Saúde da Família, Universidade Federal de Rondônia, Porto Velho, Rondônia, Brasil.

ORCID: 0000-0002-8495-966X 


\section{Karla da Silva Guimarães Rocha}

Enfermeira, pós-graduanda do Mestrado Profissional em Saúde da Família (ProfSaúde), Universidade Federal de Rondônia, Porto Velho, Rondônia, Brasil.

ORCID: 0000-0003-4127-0958

\section{Tamires dos Prazeres de Oliveira}

Enfermeira, pós-graduanda do Mestrado Profissional em Saúde da Família (ProfSaúde), Universidade Federal de Rondônia, Porto Velho, Rondônia, Brasil.

ORCID: 0000-0001-6354-3078

\section{Brysa Soares Vergotti}

Orientadora, Universidade Federal de Rondônia, Porto Velho, Rondônia, Brasil.

ORCID: 0000-0002-1460-0803

\section{Douglas Oliveira Vieira}

Nutricionista, Universidade Federal de Rondônia, Porto Velho, Rondônia, Brasil. ORCID: 0000-0002-5263-5484

\section{Kátia Fernanda Alves Moreira}

Enfermeira, Universidade Federal de Rondônia, Porto Velho, Rondônia, Brasil.

ORCID: 0000-0002-1460-0803

\section{INTRODUÇÃO}

A Atenção Primária à Saúde (APS) é considerada porta de entrada dos usuários e uma estratégia importante para reduzir as iniquidades em saúde. É entendida como o eixo norteador da Rede de Atenção à Saúde (RAS) no Sistema Único de Saúde (SUS) e responsável pela ordenação do acesso universal. Nesse contexto, as unidades básicas de saúde (UBS) devem garantir mecanismos que assegurem a acessibilidade e o acolhimento dos usuários com uma lógica organizacional ${ }^{1}$.

A doença do Coronavírus de 2019 (COVID-19), causada pelo vírus SARS-COV-2, apresentou rápida disseminação global, declarada como pandemia pela Organização Mundial da Saúde (OMS) em 11 de março de $2020^{2}$. Neste cenário, as ações da APS devem ser resolutivas às necessidades de saúde dos usuários e os processos de trabalho organizados, para o enfrentamento de doenças emergentes com alto poder de infecção.

Faz-se necessário, discutir o papel da APS no enfrentamento à pandemia, pois, segundo estudos, cerca de $80 \%$ dos casos são leves e grande parte dos casos moderados procuram a APS como primeiro acesso no cuidado 3 . Dessa forma, apesar das mudanças trazidas pela COVID-19,
Neste cenário, as ações da APS devem ser resolutivas às necessidades de saúde dos usuários e os processos de trabalho organizados, para o enfrentamento de doenças emergentes com alto poder de infecção. a APS precisa assegurar o acesso à saúde e uma cobertura da população do território de forma integral e universal ${ }^{4}$.

Partindo deste pressuposto, a pandemia de COVID-19 implicou na APS a exigência de medidas efetivas, garantindo o controle da infecção e, ao mesmo tempo, os atendimentos de gestantes, crianças, idosos, vacinação e dispensação de medicamentos de forma segura e eficaz. Para tanto, a elaboração de protocolos, fluxogramas e notas técnicas para orientar as ações dos serviços para os profissionais de saúde faz-se essencial, evitando assim, que os usuários fiquem desassistidos e provendo um acompanhamento de qualidade ${ }^{4}$.

No intuito de fortalecer a APS, aliada a necessidade de ampliar a reorientação na formação dos profissionais da saúde, surgem as Residências Multiprofissionais em Saúde (RMS), criadas a partir da promulgação da Lei Federal no 11.129/2005. As RMS são orientadas pelos princípios e diretrizes do SUS 5 e emergem como uma possibilidade concreta de articulação dos serviços de saúde com as instituições de ensino e comunidade, problematizando a realidade do território. Nesse contexto, a APS vem ocupando lugar de destaque no tocante à equidade e ao acesso universal, garantindo acessibilidade e qualidade da atenção em saúde ${ }^{6}$. 


\section{artigo}

Bragado, M.J.V.; Rocha, K.S.G.; Oliveira, T.P.; Vergotti, B.S.; Vieira, D.O.; Moreira, K.F.A.;

Organização do atendimento em saúde durante pandemia do coronavírus: o reflexo do trabalho colaborativo

Sendo assim, este estudo tem por objetivo relatar a experiência de uma residente em saúde da família (Remusf) acerca da reorganização do fluxo de atendimento em uma Unidade de Saúde da Família (USF) durante a pandemia do Coronavírus.

\section{MÉTODO}

Trata-se de um estudo descritivo, do tipo relato de experiência, acerca da vivência de uma residente em saúde da família da Fundação Universidade Federal de Rondônia (UNIR) inserida na Estratégia Saúde da Família (ESF) da USF José Adelino da Silva, no município de Porto Velho, Rondônia.

A USF situa-se na zona leste do município de Porto Velho-RO. É composta por cinco equipes de Saúde da Família (eSF) que cobrem os bairros: Ulisses Guimarães,
Marcos Freire, Airton Sena e Ronaldo Aragão. Ademais, há grande número de usuários que habitam em áreas descobertas por eSF e residem em áreas rurais.

As eSF são compostas por médicos, enfermeiros, odontólogos, técnicos de enfermagem, auxiliar de odontologia e agentes comunitários de saúde (ACS). Além de receber os profissionais da Remusf, sendo: um enfermeiro, um profissional de educação física, um farmacêutico, um fisioterapeuta e um psicólogo.

A USF dispõe de serviço de recepção (Same), serviço de regulação, farmácia, laboratório, sala de vacinação, sala de curativo, sala de procedimento, triagem, três consultórios médicos, três consultórios de enfermagem, três consultórios odontológicos e um Centro de Especialidades Odontológicas (CEO). Vale ressaltar, que em uma parte do prédio, funciona o pron-
Figura 1: Representação esquemática da reorganização do fluxo de atendimento na USF José Adelino da Silva durante a pandemia do coronavírus.
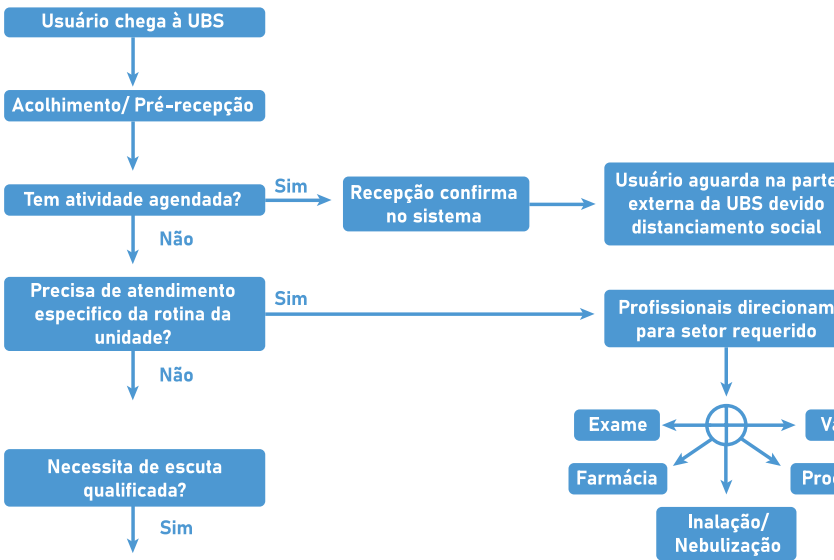

Sim
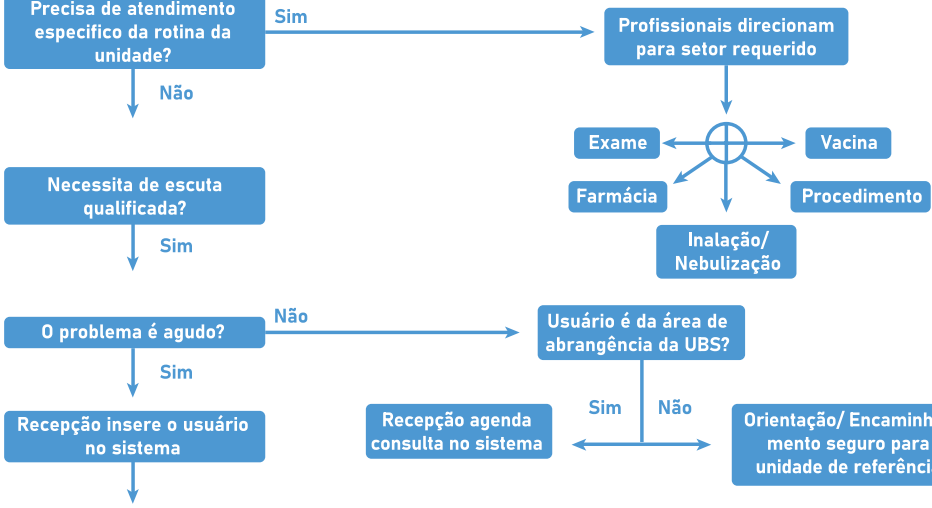

Usuá
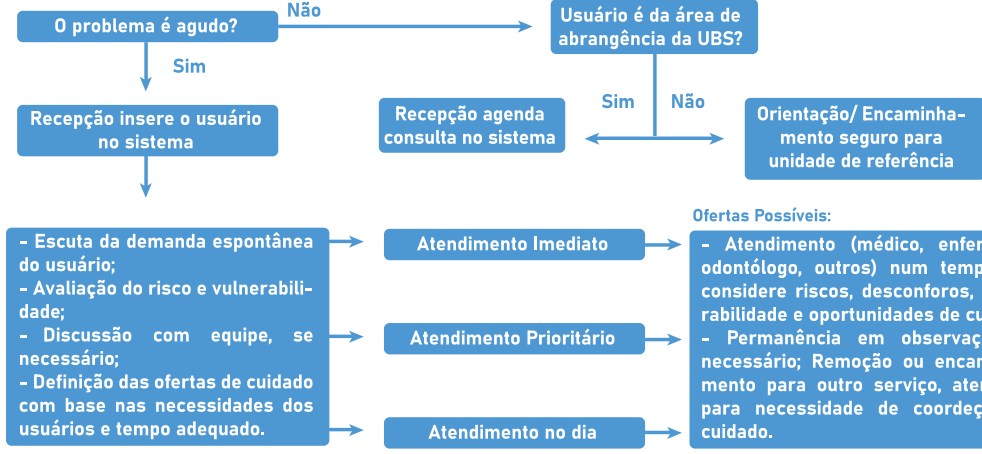

Ofertas Possiveis:

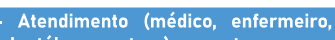

odontólogo, outros) num tempo que considere riscos, desconforos, vulnerabilidade e oportunidades de cuidado;

Permanência em observaçäo se necessário; Remoção ou encaminhamento para outro serviço, atentando para necessidade de coordeção de para neces
cuidado. to atendimento $(\mathrm{PA})$, porém, frequentemente há falta de médicos, o que implica no aumento da demanda de atendimento na USF.

Assim, o fluxo de atendimento encontrava-se desorganizado com alta demanda espontânea em detrimento da demanda programada devido ao grande número de usuários advindos de territórios descobertos de eSF. A USF encontrava-se na tentativa de adequar o fluxo de agenda programada e demanda espontânea quando iniciou a pandemia, necessitando assim, a urgente organização do atendimento.

\section{RESULTADOS E DISCUSSÃO}

A implementação do fluxo de atendimento durante a pandemia deu-se através da organização dos tipos de demandas da USF, divididas entre demandas espontâneas, programadas e procedimentos da carteira de serviços oferecidos pela USF.

O fluxo de atendimento (Figura 1) foi estruturado a partir de discussões com os profissionais da USF e residentes, considerando o plano de contingência da Secretaria Municipal de Saúde (SEMUSA) de Porto Velho- $\mathrm{RO}^{7}$.

Este processo em que vários profissionais de saúde trabalham coletivamente para impactar positivamente as práticas de cuidado é compreendido como colaboração interprofissional (PIC), que envolve negociação sistemática e interação entre profissionais, objetivando discutir as ações que serão realizadas pelos diversos atores sociais ${ }^{8}$.

O fluxo de cuidado foi estruturado visando reduzir aglomerações e manter o acesso e resolutividade da assistência. Assim, a USF organizou os seguintes cenários de cuidados: Acolhimento/pré-recepção, recepção, acolhimento com escuta qualificada, atendimento programado e telemonitoramento.

De acordo com o fluxograma (Figura 1 ), o primeiro contato entre os profissionais e usuários dar-se no acolhimento/ pré-recepção. Esta estratégia organiza-se na entrada e objetiva filtrar a demanda e direcionar o fluxo interno da USF, respei- 
tando o distanciamento social e quantidade mínima de pessoas.

O acolhimento é um importante dispositivo na organização da APS como modo de viabilizar o acesso dos usuários aos serviços de saúde?. Trata-se da "recepção do usuário, desde sua chegada, responsabilizando-se integralmente por ele, ouvindo sua queixa, permitindo que ele expresse suas preocupações e angústias e, ao mesmo tempo, colocando os limites necessários, garantindo atenção resolutiva e articulação com outros serviços de saúde para a continuidade da assistência”"10.

Dessa forma, neste setor, é questionado ao usuário quanto suas queixas e área de moradia. Assim, torna-se possível identificar se trata de um caso agudo, crônico ou crônico agudizado e estes residem em áreas cobertas por eSF, visando a garantia da longitudinalidade do cuidado. Sendo assim, os usuários que não fazem parte da área de abrangência da USF são orientados e encaminhados para sua unidade de referência. Vale destacar que, qualquer usuário com caso agudo ou crônico agudizado será atendido independente de sua unidade de referência.

$\mathrm{Na}$ recepção, dar-se continuidade ao fluxo. Uma vez identificado a necessidade do usuário, o profissional presente no acolhimento/pré-recepção solicita os documentos e cartão do SUS e dirige-se até a recepção, enquanto o usuário aguarda o atendimento na parte externa da USF, considerando a ambientação ampla e bem ventilada e evitando aglomerações.

Com o objetivo de organizar o acesso à UBS, propõe-se a classificação em: atenção à demanda espontânea- urgência/emergência e atenção à demanda programada. Quanto a primeira, as situações que não se caracterizam como urgência ou emergência deverão ter a situação/queixa avaliada pelo profissional que poderá, dependendo da sua necessidade e da disponibilidade do serviço: atendê-lo de imediato; agendar uma consulta; encaminhá-lo para outro ponto de atenção. Já na atenção programada, procurar-se-á respeitar o critério de adscrição da clientela da eSF, ou seja, o usuário deverá, preferencialmente, ser identificado pelo

ACS e atendido pelo médico, enfermeiro ou dentista de sua área?.

Sendo assim, para que haja êxito no acesso, é necessário organização do acolhimento com escuta qualificada. Diferentemente da triagem, a escuta qualificada objetiva acolher o usuário realizando uma abordagem pautada na equidade, visão ampliada do indivíduo e atenção integral.

As demandas

\section{que se enquadram}

nas cores vermelho,

amarelo e verde

devem ser

atendidas no

dia de acordo

com suas

necessidades e

tempo adequado

e, as demandas

classificadas na

cor azul terão

consultas agendadas

através de blocos

de horas
Neste cenário, realiza-se uma escuta das demandas dos usuários com classificação de risco e vulnerabilidades e, a partir disso, define-se as ofertas de cuidado.

As intervenções segundo a estratificação da necessidade do usuário, são classificá-las em "Não Agudo" (intervenções programadas) e "Agudo" (atendimento imediato, prioritário ou no dia). Sendo que, as situações não agudas e os três tipos de atendimento de situações agudas ou crônicas agudizadas foram representados por cores ${ }^{11}$.

As demandas que se enquadram nas cores vermelho, amarelo e verde devem ser atendidas no dia de acordo com suas necessidades e tempo adequado e, as demandas classificadas na cor azul terão consultas agendadas através de blocos de horas ${ }^{11}$. Já os atendimentos programados são agendados previamente em consenso com os usuários, evitando o acúmulo das pessoas nos mesmos horários e os longos períodos de espera?.

Em contrapartida, na pandemia, muitos usuários da área de abrangência não estão frequentando a UBS devido o isolamento social, angústia, medo. Isso remete uma reflexão a respeito do acesso, longitudinalidade e responsabilização com o território adscrito. Dessa forma, valorizando os objetivos e diretrizes do SUS, implantou-se o Telemonitoramento.

O telemonitoramento caracteriza-se pelo acompanhamento a distância dos pacientes, seja para avaliação de tratamento ou para verificação epidemiológica. Embora essa prática não fosse utilizada em larga escala pelo SUS, constituiu uma importante estratégia para favorecer o distanciamento social e ao mesmo tempo, a continuidade da assistência ${ }^{12}$.

Dessa forma, através da busca no território, os ACS identificam os usuários com $S G$, bem como, outras queixas e necessidades. A partir disso, as eSF efetuam monitoramento via telefone e, caso necessário, intervenções na unidade ou domicílio.

Verifica-se que a USF, além de buscar atender os usuários durante a pandemia, também procura implementar reflexões sobre as competências específicas e comuns 
por meio da Educação Interprofissional (EIP) que, segundo Costa ${ }^{13}$ potencializam o desenvolvimento das competências colaborativas, uma vez que, favorecem a interação entre os profissionais.

\section{CONCLUSÃO}

A reorganização dos fluxos de atendimento na APS somado aos esforços para enfrentamento da pandemia, evidenciou a potencialidade do trabalho na perspectiva interprofissionalidade. Foi possível implantar o acolhimento, escuta qualificada, agenda programada e não foram observados aglomerações e filas durante os atendimentos.

A participação dos residentes trouxe uma nova dinâmica no processo ensino-
Foi possível implantar

o acolhimento, escuta

qualificada, agenda

programada e não

foram observados

aglomerações e

filas durante os

atendimentos -aprendizagem e viabilizou a integração entre serviço e ensino e o desenvolvimento de práticas colaborativas. Além disso, os momentos de discussões dos fluxos entre as equipes propiciaram resgatar o papel de cada profissional inserido na APS, ressignificando e fortalecendo o trabalho em equipe, a eficácia e a efetividade da assistência e dos cuidados de saúde.

Por fim, espera-se por este estudo, disseminar as potencialidades da construção coletiva, considerando diferentes perspectivas e o olhar interprofissional sobre temas transversais às profissões e que, motive os profissionais da saúde a investirem e aplicarem a EIP em diferentes áreas do conhecimento.

\section{REFERÊNCIAS}

1. Brasil. Ministério da Saúde. Secretaria de Atenção à Saúde. Departamento de Atenção Básica. Acolhimento à demanda espontânea / Ministério da Saúde. Secretaria de Atenção à Saúde. Departamento de Atenção Básica. - 1. ed.; 1. reimpr. - Brasília: Ministério da Saúde, 2013. 56 p.: il. - (Cadernos de Atenção Básica; n. 28, V. 1).

2. World Health Organization (WHO). WHO Director-General's opening remarks at the media briefing on COVID-19 - 11 March 2020 [Internet]. Geneve: WHO; 2020 [cited 2020 Mar 23]. Available from: https://www.who.int/dg/speeches/detail/whodirector-general-s-opening-remarks-at-the-media-briefing-oncovid-19---11-march-2020.

3. Dunlop C, Howe A, Li D, Allen LN. The coronavirus outbreak: the central role of primary care in emergency preparedness and response. BJGP Open [Internet]. 2020 Jan [cited $2020 \mathrm{Apr}$ 15]:pii:bjgpopen20X101041. Available from:

https://doi.org/10.3399/bjgpopen20X101041.

4. Andres SC, Carlotto $A B$, Leão $A$. A organização e estruturação do serviço de saúde na APS para o enfretamento da Covid-19: relato de experiência. 1 [Internet]. 10 de abril de 2021 [citado 10 de maio de 2021];3(1):09-15. Disponível em: https://apsemrevista.org/aps/article/view/137

5. Brasil. Lei $n^{\circ}$ 11.129, 30 de junho de 2005. Institui o Programa Nacional de Inclusão de Jovens - Projovem; cria o Conselho Nacional da Juventude (CNJ) e a Secretaria Nacional da Juventude; altera as Leis $n^{\circ} 10.683$, de 28 de maio de 2003, e 10.429, de 24 de abril de 2002; e dá outras providências. Diário Oficial da União, Brasília, DF, 30 jun. 2005.

6. Ribeiro JP, Mamed SN, Souza MR, Souza MM, Rosso CFW. Acessibilidade aos serviços de saúde na Atenção Básica do Estado de Goiás. Rev. Eletr. Enf, Goiania, v.17, n.3, p. 1-11, jul./set. 2015.
7. Prefeitura do município de Porto Velho. Secretaria municipal de saúde. Plano municipal de contingência para resposta às emergências em saúde pública coronavírus. Porto Velho, abril, 2020.

8. Reeves S, Pelone F, Harrison R, Goldman J, Zwarenstein M. Interprofessional collaboration to improve professional practice and healthcare. 102 outcomes. Cochrane Database of Systematic Reviews 2017, Issue 6. Art. No.: CD000072. DOI: 10.1002/14651858.CD000072.pub3.

9. BRASIL. Conselho Nacional de Secretários de Saúde. Planificação da Atenção Primária à Saúde nos Estados / Conselho Nacional de Secretários de Saúde. - Brasília: CONASS, 2011.

10. Brasil. Ministério da Saúde. Secretaria de Atenção à Saúde. Núcleo Técnico da Política Nacional de Humanização. HumanizaSUS : documento base para gestores e trabalhadores do SUS / Ministério da Saúde, Secretaria de Atenção à Saúde, Núcleo Técnico da Política Nacional de Humanização. - 3. ed. - Brasília: Editora do Ministério da Saúde, 2006.

11. Brasil. Ministério da Saúde. Secretaria de Atenção à Saúde. Departamento de Atenção Básica. Acolhimento à demanda espontânea: queixas mais comuns na Atenção Básica / Ministério da Saúde, Secretaria de Atenção à Saúde, Departamento de Atenção Básica. - Brasília: Ministério da Saúde, 2012. 290 p.: il. - (Cadernos de Atenção Básica n. 28, Volume II).

12. Santos GG, Paco JAO. Visão e atuação humanizada de estudante do curso de Especialização em Enfermagem Obstétrica no parto. Glob Acad Nurs. 2020;1(1):e9. https://dx.doi. org/10.5935/2675-5602.20200009.

13. Costa MV. A educação interprofissional no contexto brasileiro: algumas reflexões. Interface (Botucatu), v. 20, no. 56, pp. 97-198, 2016. Disponível em: http://www.scielo.br/scielo. php?script=sci_arttext\&pid=S1414-32832016000100197. 